\title{
C1QBP is upregulated in colon cancer and binds to apolipoprotein A-I
}

\author{
KUN KIM $^{1,2^{*}}$, MIN-JEONG KIM ${ }^{3 *}$, KYUNG-HEE KIM $^{1}$, SUN-A AHN $^{1}$, \\ JONG HEON KIM ${ }^{4}$, JAE YOUL CHO ${ }^{5}$ and SEUNG-GU YEO ${ }^{6}$ \\ ${ }^{1}$ Colorectal Cancer Branch, Research Institute, National Cancer Center, Goyang, Gyeonggi 10408; \\ ${ }^{2}$ Laboratory of Cell Biology, Cancer Research Institute, Seoul National University, Seoul 03080; \\ ${ }^{3}$ Department of Radiology, Hallym Sacred Heart Hospital, Hallym University College of Medicine, Anyang, Gyeonggi 14068; \\ ${ }^{4}$ Cancer Cell and Molecular Biology Branch, Research Institute, National Cancer Center, Goyang, Gyeonggi 10408; \\ ${ }^{5}$ Department of Genetic Engineering, Sungkyunkwan University, Suwon, Gyeonggi 16419; ${ }^{6}$ Department of Radiation \\ Oncology, Soonchunhyang University College of Medicine, Soonchunhyang University Hospital, \\ Cheonan, South Chungcheong 31151, Republic of Korea
}

Received September 30, 2015; Accepted October 28, 2016

DOI: $10.3892 /$ etm.2017.4249

\begin{abstract}
The present study aimed to investigate the expression of complement component 1, q subcomponent-binding protein $(\mathrm{C} 1 \mathrm{QBP})$ in colon cancer cells, and identify proteins that interact with C1QBP. Total proteins were extracted from both the tumor and normal tissues of 22 patients with colon cancer and analyzed using liquid chromatography-mass spectrometry (LC-MS) to identify proteins that were differentially-expressed in tumor tissues. C1QBP overexpression was induced in $293 \mathrm{~T}$ cells using a pFLAG-CMV2 expression vector. Overexpressed FLAG-tagged C1QBP protein was then immunoprecipitated using anti-FLAG antibodies and C1QBP-interacting proteins were screened using LC-MS analysis of the immunoprecipitates. The C1QBP-interacting proteins were confirmed using reverse-immunoprecipitation and the differential expression of C1QBP in tissues and cell lines was confirmed using western blot analysis. LC-MS analysis revealed that C1QBP exhibited a typical tumor expression pattern. Two immune-reactive signals (33 and $14 \mathrm{kDa}$ ) were detected in normal and tumor tissues from 19 patients. Furthermore, 14 kDa C1QBP protein was upregulated in the tumors of 15 patients. In total, 39 proteins were identified as candidate C1QBP-interacting proteins, and an interaction between C1QBP and apolipoprotein A-I was
\end{abstract}

Correspondence to: Dr Seung-Gu Yeo, Department of Radiation Oncology, Soonchunhyang University College of Medicine, Soonchunhyang University Hospital, 31 Soonchunhyang 6-gil, Cheonan, South Chungcheong 31151, Republic of Korea

E-mail: md6630@schmc.ac.kr

*Contributed equally

Key words: complement component 1, q subcomponent-binding protein, colon cancer, apolipoprotein A-I, proteomics confirmed. The present study indicates that $\mathrm{C} 1 \mathrm{QBP}$ is involved in colon cancer carcinogenesis, and that the mechanisms underlying the established anti-tumor properties of apolipoprotein A-I may include interacting with and inhibiting the activity of C1QBP.

\section{Introduction}

Complement component 1, q subcomponent binding protein (C1QBP) is a multifunctional protein. Its sequence is identical to that of $\mathrm{p} 32$, a protein that co-purifies with splicing factor SF2, and that of hyaluronan-binding protein 1 , a member of the hyaladherin family (1). Its terminal $\alpha$-helices are important in protein-protein interactions and regulate its multifunctional nature. C1QBP is commonly localized in the mitochondrial matrix, although it exhibits differential localization among cell lines under various physiological conditions (1). Moreover, it serves important roles in various biological processes, including inflammation, infection, cell signaling and chemotaxis (2-4).

However, it remains unclear whether C1QBP exerts antior pro-tumorigenic effects during cancer pathogenesis (5). Upregulated expression of $\mathrm{C} 1 \mathrm{QBP}$ has been reported in various different types of cancer, including breast, prostate, thyroid, colon, pancreatic, gastric, esophageal and lung cancer (6-9). This elevated expression has been linked to the metastasis of ovarian cancer (10), poor prognosis or metastasis of breast cancer $(7,9)$ and early relapse following surgery in prostate cancer (11). By contrast, other studies have reported that C1QBP is required for the induction of mitochondria-dependent apoptotic cell death $(12,13)$. The constitutive expression of C1QBP in normal fibroblast cells perturbs its growth characteristics and induces apoptosis (14). In addition, it has been demonstrated that C1QBP is upregulated following the induction of apoptosis in HeLa cells in response to a chemotherapeutic agent (cisplatin), suggesting that C1QBP may be involved in apoptosis $(15,16)$. 
Over one million people worldwide develop colorectal cancer annually, and the disease has a mortality rate of $\sim 33 \%$ (17). Carcinogenesis and biology of colorectal cancer have been recognized as multistep processes involving various molecular alterations (18). Knowledge of the specific molecular markers or pathways that are responsible for disease progression and poor prognosis may be beneficial in the development of more effective treatment.

The present proteomic study revealed that C1QBP was cleaved in the tumor tissues of patients with colon cancer, and that apolipoprotein A-I (ApoA-I) interacts with C1QBP. Furthermore, the possible role of C1QBP in colon cancer via its interaction with ApoA-I is discussed.

\section{Materials and methods}

Tissues from colorectal cancer patients. Fresh tissues (normal colon mucosa and primary colon tumor) were obtained from 22 patients with colon cancer ( 8 female and 14 male, $61.5 \pm 10.5$ years old) who were enrolled in the current study between January 2006 and May 2007 at the National Cancer Center, Republic of Korea. Following dissection of necrotic exudates and stromal components, the overall cellularity of the normal epithelium and tumors was $>75 \%$. The present study was approved and performed in accordance with the guidelines of the Institutional Review Board of the National Cancer Center (Goyang, Republic of Korea), and informed consent was obtained from all patients.

Human cancer cell lines. The human colon cancer cell lines (SNU-81, SNU-407, SNU-C4, NCI-H498, NCI-H508, CaCo2, DLD-1, HCT-116, LoVo and SW620), a cervical cancer cell line (HeLa) and an embryonic kidney cell line (293T) were all obtained from the Korean Culture Line Bank (Seoul, Korea).

Mass analysis. Mass analysis was performed as described previously (19). The bands of SDS-PAGE corresponding to potential proteins of interest were excised, destained using $50 \%$ acetonitrile in $0.1 \mathrm{M}$ ammonium bicarbonate (Thermo Fisher Scientific, Inc., Waltham, MA USA) and dried using a SpeedVac evaporator (Savant SVC-100H, Thermo Fisher Scientific, Inc.). Dried gel pieces were swollen with $30 \mu 1$ of $25 \mathrm{mM}$ sodium bicarbonate (pH 8.8) (Thermo Fisher Scientific, Inc.) containing $50 \mathrm{ng}$ trypsin (Promega Corporation, Madison, WI, USA) at $37^{\circ} \mathrm{C}$ overnight. Samples were subsequently desalted using Zip-Tips C18 (EMD Millipore, Billerica, MA, USA) and dissolved in $10 \mu 1$ of $2 \%$ acetonitrile (Thermo Fisher Scientific, Inc.) in $0.1 \%$ formic acid (Thermo Fisher Scientific, Inc.). Analyses were performed using a linear trap quadrupole (LTQ) XL linear ion trap mass spectrometer (MS; Thermo Fisher Scientific, Inc.) in the Proteomics Core of the National Cancer Center (Goyang, Republic of Korea). The mass spectrometer was set for nanospray ionization (NSI) in the positive mode. Moreover, a syringe pump was used to introduce the calibration solution for the automatic tuning and calibration of the LTQ in an NSI-positive ion mode. The infusion of trypsin-digested samples into the ionization source of the mass spectrometer was performed following liquid chromatographic separation. The spray voltage was set at $+1.1 \mathrm{kV}$, the temperature of the capillary apparatus was maintained at $200^{\circ} \mathrm{C}$, the capillary voltage was set at $+20 \mathrm{~V}$ and the tube lens voltage was $+100 \mathrm{~V}$. Moreover, the auxiliary gas was set to zero. Full scan experiments were performed to linear trap in the $\mathrm{m} / \mathrm{z}$ range of $150-2,000$. Systematic MS/MS experiments were performed by changing the relative collision energy and monitoring the intensities of the fragment ions. All MS/MS samples were analyzed using Sequest (version v.27, rev.11; Thermo Fisher Scientific) to search the uniprot_ sprot (http://www.uniprot.org) and IPI human databases (European Bioinformatics Institute, Hinxton, UK; ftp://ftp. ebi.ac.uk/pub/databases/IPI/last_release/current/) assuming digestion with trypsin. Sequest was searched with a fragment ion mass tolerance of $1.00 \mathrm{Da}$ and a parent ion tolerance of 1.2 Da. Methionine oxidation was specified as a variable modification.

Western blot analysis. Western blotting was performed as described previously (20). Briefly, cell homogenates containing equivalent quantities $(20 \mu \mathrm{g})$ of protein were centrifuged at $4,000 \times g\left(4^{\circ} \mathrm{C}\right)$ for $5 \mathrm{~min}$ and the supernatant fractions were separated using 4-10\% gradient SDS-PAGE. Following electrophoresis, the proteins were transferred to polyvinylidene fluoride membranes (EMD Millipore), blocked for $2 \mathrm{~h}$ at $4^{\circ} \mathrm{C}$ in $1 \%$ Tween 20 -Tris buffered saline buffer containing $1.5 \%$ non-fat dry milk (Bio-Rad Laboratories, Inc., Hercules, CA, USA) and $1 \mathrm{mM} \mathrm{MgCl}_{2}$. The blocked membranes were then incubated for $2 \mathrm{~h}$ at room temperature with primary antibodies against C1QBP (dilution 1:1,000, cat no. ab131284, Abcam, Cambridge, MA, USA), ApoA-I (dilution 1:1,000, cat no. ab52945, Abcam) or $\beta$-actin (dilution 1:2,000, cat no. 04-1116, Merck Millipore, Darmstadt, Germany). They were then washed three times in blocking solution (1\% Tween 20-TBS buffer containing 1.5\% non-fat dry milk and $1 \mathrm{mM} \mathrm{MgCl}$ ) for 15 min each and incubated with diluted horseradish-peroxidase conjugated secondary antibody (dilution 1:2,000, cat no. 4010-05, Southern Biotech Associates, Inc., Birmingham, AL, USA) for $1 \mathrm{~h}$ at room temperature. Membranes were then washed with blocking solution $(3 \times 15 \mathrm{~min})$, incubated with WEST-ZOL ${ }^{\circledR}$ plus chemiluminescence reagent (iNtRON Biotechnology, Inc., Gyeonggi, Korea) for $1 \mathrm{~min}$ and exposed to film (Kodak Blue XB-1, Kodak, Rochester, NY, USA).

Overexpression of ClQBP in 2937 cells and immunoprecipitation. In order to generate a pFLAG-CMV2-C1QBP plasmid containing the full-length coding sequence of human $C 1 Q B P$, polymerase chain reaction (PCR) was performed from a human brain cDNA library (Clontech Laboratories, Inc., Rochester, NY, USA) with the following oligomers: Sense, 5'-GGAATTCTATGCTGCCTCTGCTGCGCTGC-3' and antisense, 5'-TAACCCGGGCTACTGGCTCTTGACAAA ACTCTTGAGG-3'. The amplified PCR product was digested using EcoRI-XmaI (Clontech Laboratories), then inserted into pFLAG-CMV2 (Merck Millipore). The 293T cells were transfected with control or FLAG-C1QBP plasmids. At $48 \mathrm{~h}$ after transfection, cells were harvested and aliquots of the total protein from each sample were analyzed using western blotting, as indicated.

For immunoprecipitation-coupled MS analyses, cells were lysed in immunoprecipitation buffer containing $150 \mathrm{mM} \mathrm{NaCl}$, 
$25 \mathrm{mM}$ HEPES-KOH (pH 7.5), 10\% (v/v) glycerol, $1 \mathrm{mM}$ $\mathrm{MgCl}_{2}, 2 \mathrm{mM}$ sodium orthovanadate, $2 \mathrm{mM}$ glycerophosphate, 1 mM PMSF, 1 mM DTT, 2 mM EDTA, 0.5\% Triton X-100 and $1 \mathrm{X}$ protease inhibitor cocktail (Roche Applied Science, Madison, WI, USA). Following a brief sonication and incubation on ice, the lysates were centrifuged at $15,000 \times \mathrm{x}$ for $5 \mathrm{~min}$ to remove the insoluble materials. The lysates were then incubated with anti-FLAG M2 affinity agarose beads (Sigma-Aldrich; Merck Millipore) for $2 \mathrm{~h}$ at $4^{\circ} \mathrm{C}$, and the collected beads were washed four times with washing buffer (0.05\% Triton X-100 immunoprecipitation buffer without a protease inhibitor cocktail), and boiled in SDS sample buffer for protein immunoprecipitation-coupled MS analysis.

\section{Results}

Differential expression of CIQBP in tissues from colon cancer patients. Whole proteins were extracted from both the tumor and normal tissues of three patients and were separated by SDS-PAGE (Fig. 1). Sections of gel containing proteins were then cut according to their molecular weight and subjected to proteomic analysis. Proteins of $19-35-\mathrm{kDa}$ were analyzed using the linear ion trap MS system, which identified C1QBP to be present only in tumor tissues (Fig. 1 and Table I).

Western blot analysis was then used to confirm the expression of C1QBP in normal and tumor tissues from patients with colon cancer (Fig. 2A). Two immunoreactive signals at 33 and $14 \mathrm{kDa}$ were detected using the anti-C1QBP antibody (Fig. 2A). In contrast to the proteomic analysis, $33 \mathrm{kDa}$ C1QBP exhibited no typical expression pattern in tumor tissues (Fig. 2A). However, among the 19 pairs of tissues analyzed, 15 showed upregulation of $14 \mathrm{kDa}$ C1QBP in tumor compared with normal tissues (Fig. 2A). In human colon cancer cell lines, the expression of $33 \mathrm{kDa} C 1 \mathrm{QBP}$ was variable, whereas the short form was not detected (Fig. 2B).

Candidate CIQBP-interacting proteins. In order to further evaluate the role of C1QBP in colon cancer at the molecular level, C1QBP-interacting proteins were screened for in immunoprecipitates from FLAG-tagged C1QBP-overexpressing 293 T cells. Fig. 3 presents the results of immunoprecipitation using FLAG antibodies in whole cell lysates from C1QBP-overexpressing 293T cells (pFLAG_CMV2_C1QBP). FLAG-tagged C1QBP was immunoprecipitated successfully using anti-FLAG antibodies, and was detected as a band at $33 \mathrm{kDa}$ on the SDS-PAGE gel (Fig. 3). As a control, immunoprecipitation was performed using 293T cells overexpressing FLAG alone (pFLAG_CMV2_MOCK_293T). The appropriate bands were excised from the gels as described in Fig. 3 and the proteins therein were subjected to in-gel tryptic digestion and MS. The proteins that co-precipitated with C1QBP are listed in Table II after exclusion of proteins also identified in the control lane. In total 39 proteins, including fibrinogen $\alpha$-, $\beta$ - and $\gamma$-chains, complement C3, complement C4-A, ApoA-I and ApoA-II, were identified as possible C1QBP-interacting proteins (Table II).

Interaction between CIQBP and ApoA-I. Reverse immunoprecipitation was subsequently used to confirm the interaction between C1QBP and candidate proteins. Among the proteins

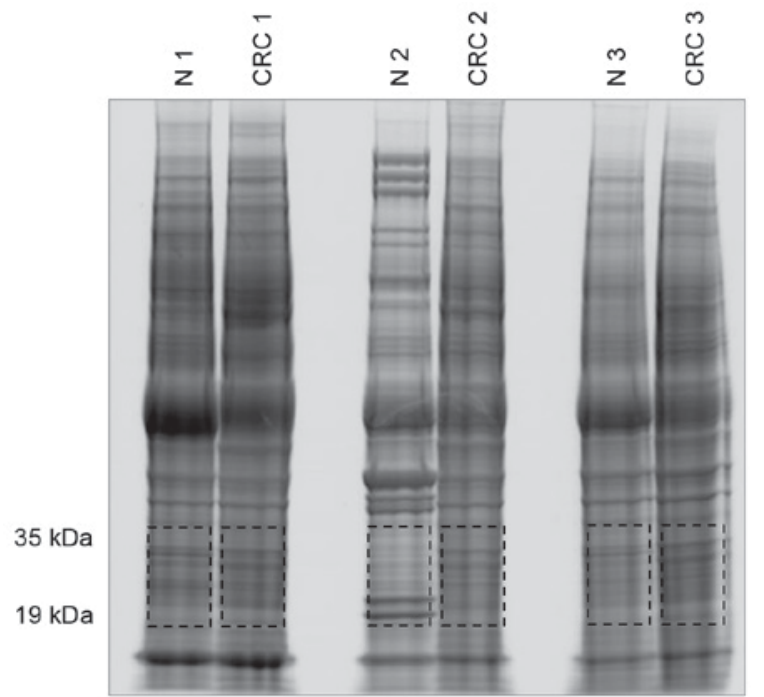

Figure 1. Image of an SDS-PAGE gel containing whole proteins extracted from paired tumor (CRC 1-3) and normal (N 1-3) tissues of three colon cancer patients. Red boxes indicate the gel slices used for mass analysis to identify proteins of 19-35 kDa.
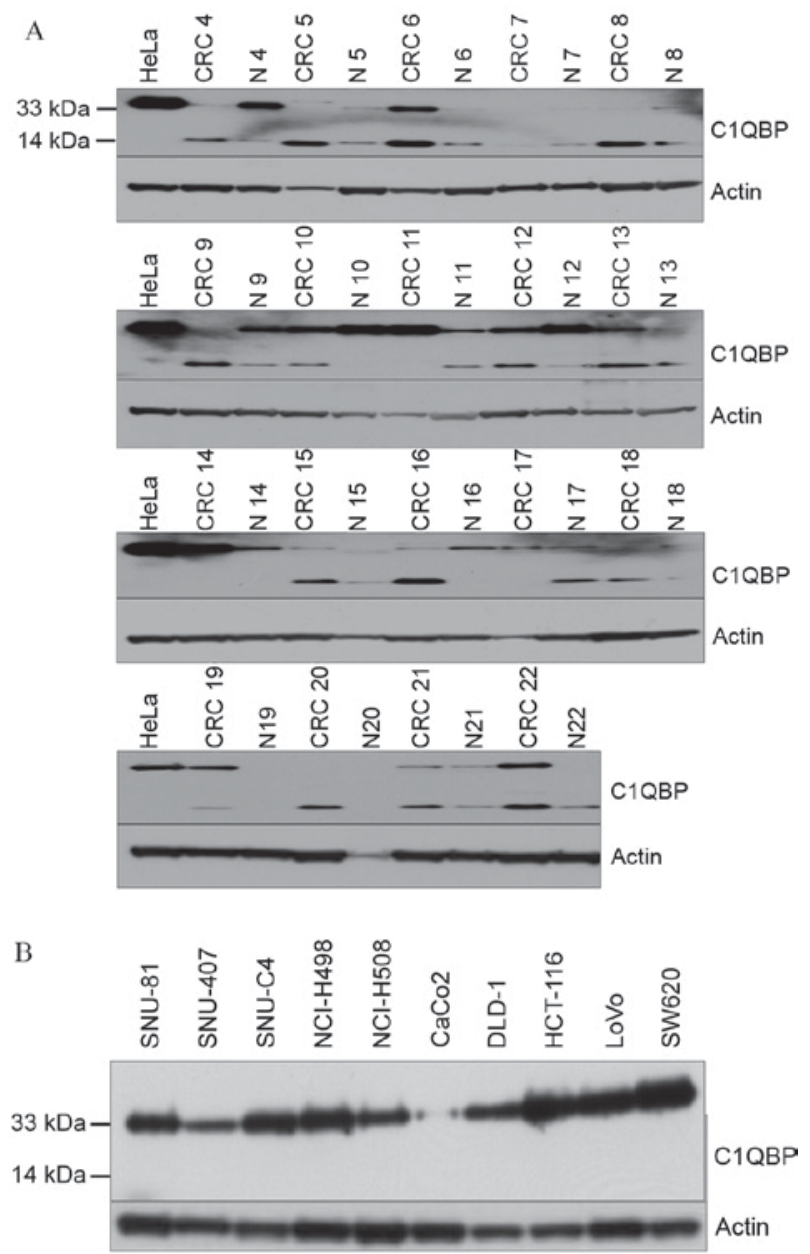

Figure 2. Expression of C1QBP in tissues from colon cancer patients and colon cancer cell lines. (A) Immunoreactive signals were detected at 33 and $14 \mathrm{kDa}$ in tissues from colon cancer patients. There was no typical expression pattern of C1QBP in tumor tissues, but increased expression of $14 \mathrm{kDa}$ C1QBP was found in 15 tumors. (B) Only $33 \mathrm{kDa}$ C1QBP was detected in human colon cancer cell lines. C1QBP, complement component 1, q subcomponent-binding protein. 


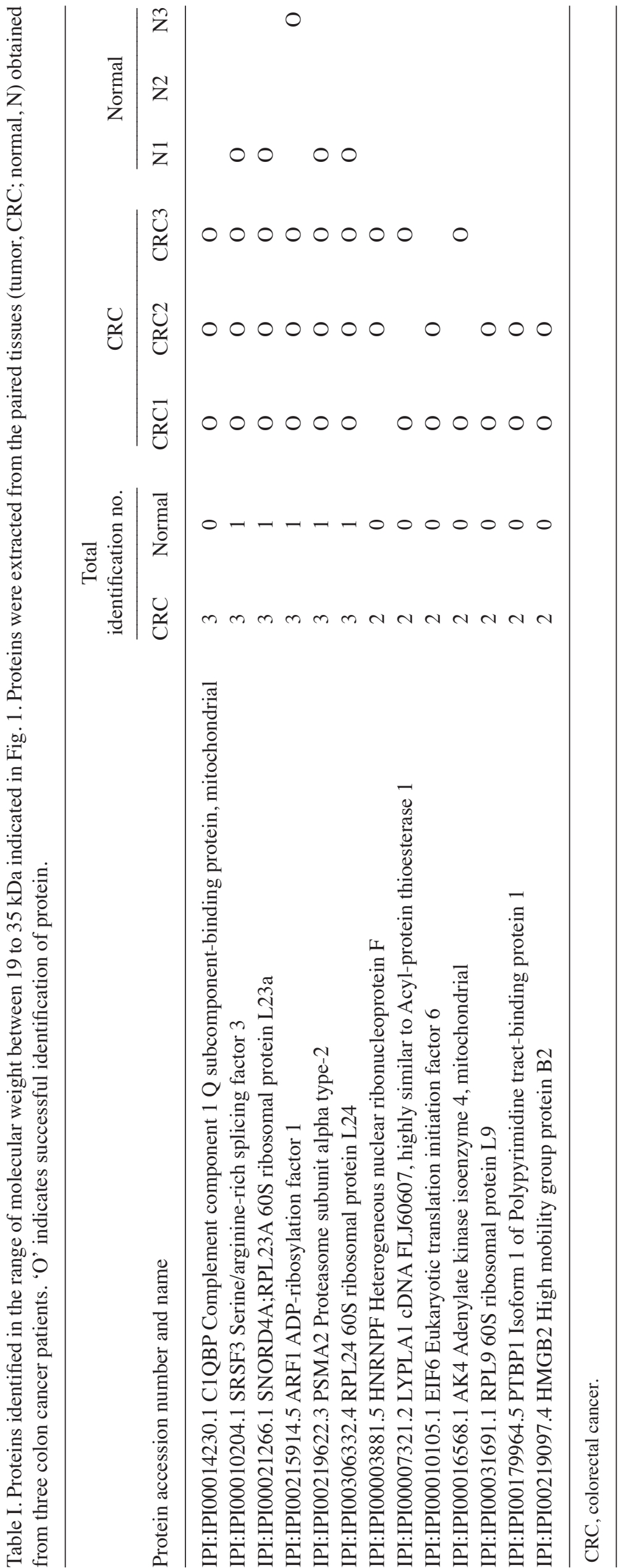




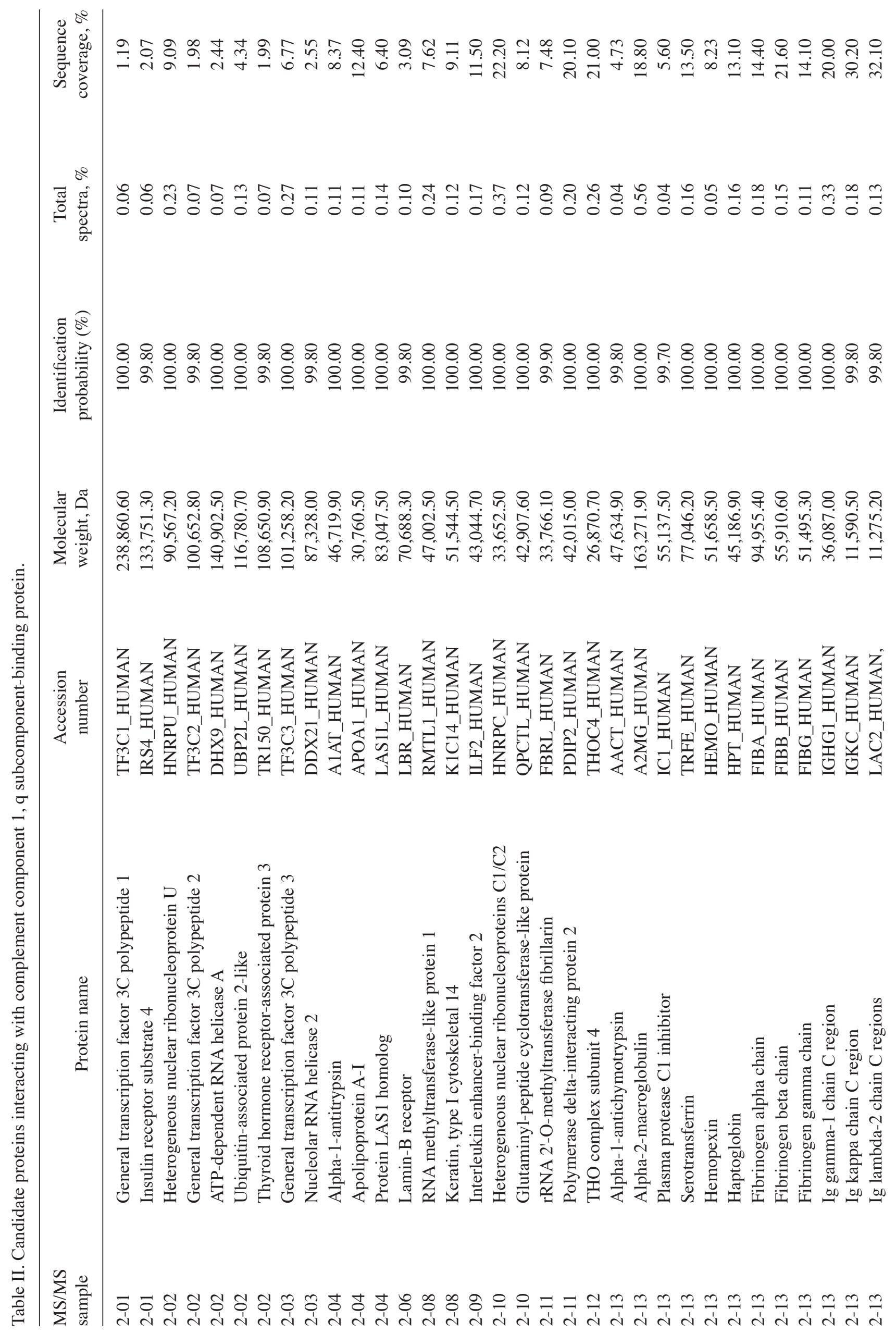



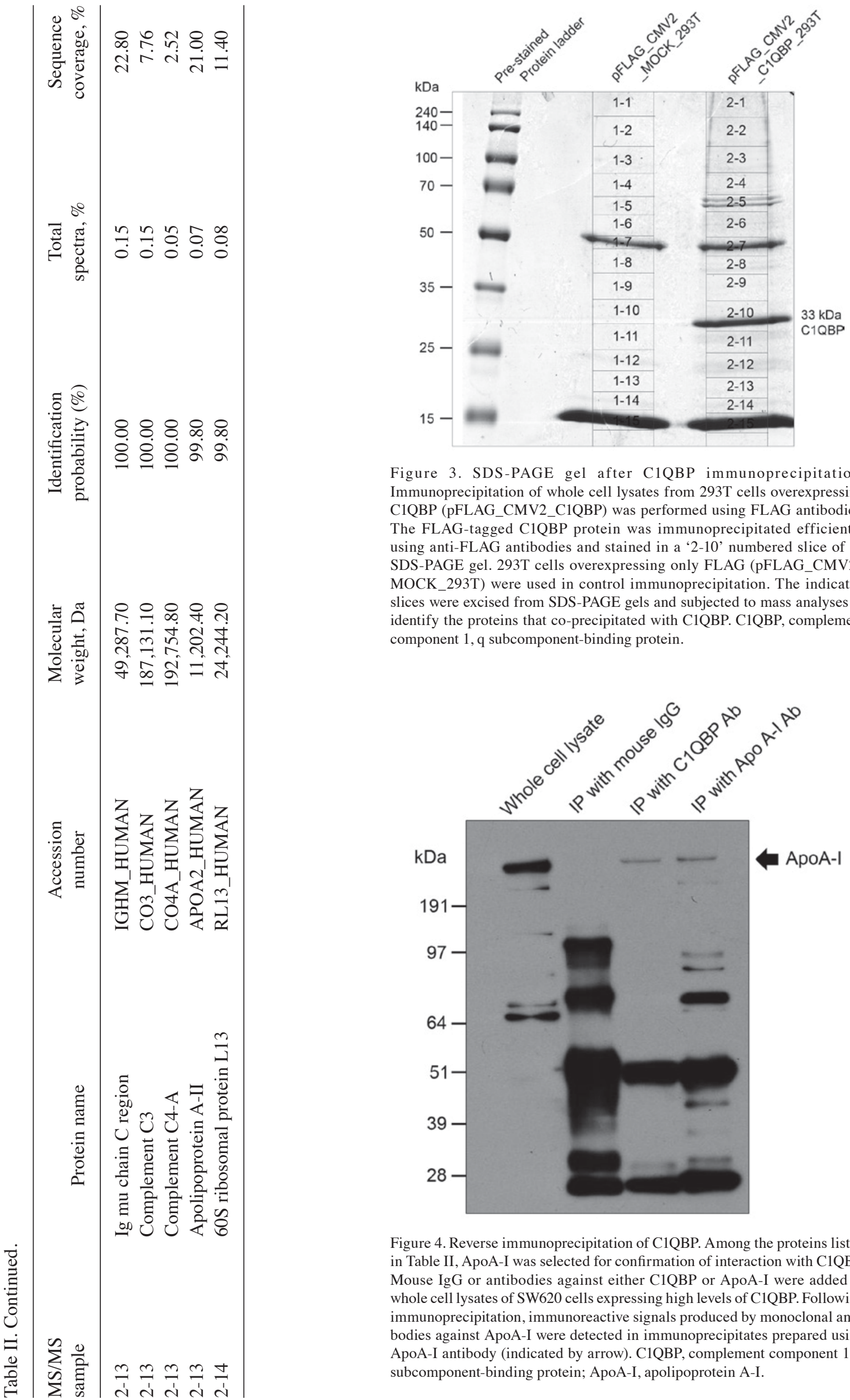

Figure 3. SDS-PAGE gel after C1QBP immunoprecipitation. Immunoprecipitation of whole cell lysates from 293T cells overexpressing C1QBP (pFLAG_CMV2_C1QBP) was performed using FLAG antibodies. The FLAG-tagged C1QBP protein was immunoprecipitated efficiently using anti-FLAG antibodies and stained in a '2-10' numbered slice of an SDS-PAGE gel. 293T cells overexpressing only FLAG (pFLAG_CMV2_ MOCK_293T) were used in control immunoprecipitation. The indicated slices were excised from SDS-PAGE gels and subjected to mass analyses to identify the proteins that co-precipitated with C1QBP. C1QBP, complement component 1 , q subcomponent-binding protein.

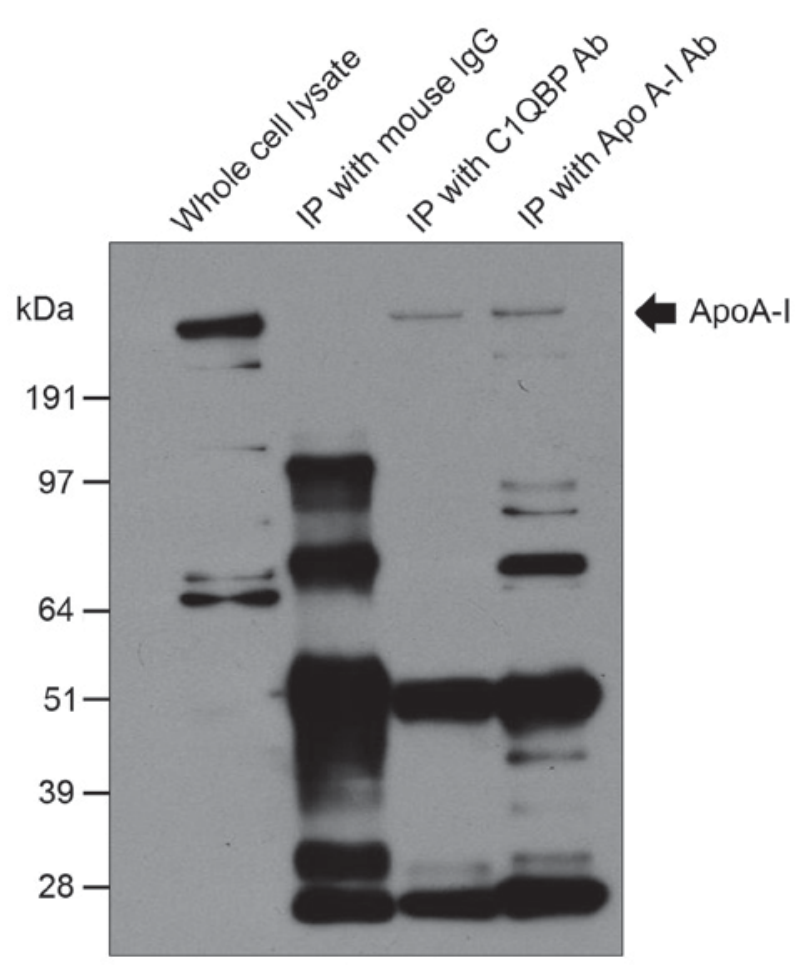

Figure 4. Reverse immunoprecipitation of C1QBP. Among the proteins listed in Table II, ApoA-I was selected for confirmation of interaction with C1QBP. Mouse IgG or antibodies against either C1QBP or ApoA-I were added to whole cell lysates of SW620 cells expressing high levels of C1QBP. Following immunoprecipitation, immunoreactive signals produced by monoclonal antibodies against ApoA-I were detected in immunoprecipitates prepared using ApoA-I antibody (indicated by arrow). C1QBP, complement component $1, \mathrm{q}$ subcomponent-binding protein; ApoA-I, apolipoprotein A-I. 
listed in Table II, ApoA-I was selected for confirmation of its interaction with C1QBP. Human SW620 colon cancer cells were used because they express high levels of C1QBP (Fig. 2B, and mouse IgG or antibodies against C1QBP and ApoA-I were added to SW620 whole-cell lysates. Immunoprecipitation was then performed, followed by western blotting. Immunoreactive signals produced by monoclonal antibodies against ApoA-I were detected in immunoprecipitates prepared using both anti-ApoA-I and -C1QBP antibodies (Fig. 4). In addition, SW620 whole cell lysates were immunoreactive for ApoA-I (Fig. 4).

\section{Discussion}

The present study revealed that the cleaved form of C1QBP (14 $\mathrm{kDa})$ is upregulated in colon cancer compared with normal cells, which is consistent with previous reports of the pro-tumorigenic properties of C1QBP $(5,6,8)$. Rubinstein et al (8) compared the C1QBP protein levels among several adenocarcinomas. Immunohistochemical staining of histological tissue sections revealed pronounced differences in the expression in colon adenocarcinoma (as well as thyroid, pancreatic, gastric, esophageal and lung cancer) vs. non-malignant tissues. Dembitzer et al (6) revealed strong C1QBP expression in epithelial breast, prostate, liver, lung, colon and skin tumors. However, increased C1QBP staining was also detected in inflammatory and proliferative lesions of the same cell types, as well as in normal and continuously dividing cells. Moreover, McGee et al (5) reported that C1QBP expression was upregulated in breast, colon and lung cancer compared with normal control tissues. Altogether, these data indicate that $\mathrm{C} 1 \mathrm{QBP}$ is important in colon cancer tumorigenicity.

A novel observation of the present study is that C1QBP interacts with ApoA-I. Among the many candidate-binding proteins identified, ApoA-I was selected for confirmation because it has a relatively well-established role in colon cancer. The lipid metabolism is closely associated with cancer $(21,22)$. In particular, it has been speculated that lipids and lipoproteins are associated with neoplastic processes, including inflammation, oxidative stress and insulin resistance (23). Moreover, ApoA-I is a major component of high-density lipoprotein in plasma, which exerts protective anti-inflammatory, anti-oxidant and anti-microbial functions, and it is important in innate immunity (21). ApoA-I is synthesized primarily in the liver (80\%) and small intestine (10\%) (24), and it is known to be important in reverse cholesterol transport and for promoting cholesterol efflux from tissues by acting as a cofactor for lecithin cholesterol acyltransferase (24). In a study of a cohort of $>520,000$ participants from 10 European countries, high serum concentrations of HDL and ApoA-I were associated with a decreased risk of colon cancer (23). In addition, HDL mimetics inhibited tumor development in both induced and spontaneous mouse models of colon cancer, possibly by inhibiting angiogenesis (21). Zhang et al (25) analyzed the lipid levels of 206 patients with colorectal cancer, 70 patients with benign colorectal disease, and 300 healthy participants, and revealed that serum ApoA-I and ApoB levels were significantly lower in colorectal cancer patients (25). In addition to colon cancer, ApoA-I inhibited tumor development in a mouse model of ovarian cancer (26). Significantly decreased serum levels of ApoA-I were found in patients with cholangiocarcinoma (27), and increased levels in serum were associated with a favorable prognosis in patients with metastatic nasopharyngeal carcinoma (28). These anti-tumor properties of ApoA-I may be associated with its binding to and subsequent inhibition of C1QBP. As such, HDL has received attention as a promising therapeutic strategy for colon cancer. A novel finding in the present study, that $\mathrm{C} 1 \mathrm{QBP}$ binds to ApoA-I, may assist the future development of such therapeutic strategies.

Nevertheless, the physiological role of the interaction between ApoA1 and C1QBP requires further investigation. Furthermore, one contradictory study indicated that the expression of ApoA-I was associated with colon adenocarcinoma progression, and that ApoA-I is a potential marker of tumor aggression (29). However, the novel observations in the present study may facilitate identification of the molecular mechanisms underlying the roles of ApoA-I and C1QBP in colon cancer.

\section{Acknowledgements}

The present study was supported by the Soonchunhyang University Research Fund.

\section{References}

1. Chowdhury AR, Ghosh I and Datta K: Excessive reactive oxygen species induces apoptosis in fibroblasts: Role of mitochondrially accumulated hyaluronic acid binding protein 1 (HABP1/p32/gC1qR). Exp Cell Res 314: 651-667, 2008.

2. Majumdar M, Meenakshi J, Goswami SK and Datta K: Hyaluronan binding protein 1 (HABP1)/C1QBP/p32 is an endogenous substrate for MAP kinase and is translocated to the nucleus upon mitogenic stimulation. Biochem Biophys Res Commun 291: 829-837, 2002.

3. Ghebrehiwet B and Peerschke EI: cClq-R (calreticulin) and gC1q-R/p33: Ubiquitously expressed multi-ligand binding cellular proteins involved in inflammation and infection. Mol Immunol 41: 173-183, 2004.

4. Tahtouh M, Garçon-Bocquet A, Croq F, Vizioli J, Sautière PE, Van Camp C, Salzet M, Nagnan-le Meillour P, Pestel J and Lefebvre C: Interaction of $\mathrm{HmClq}$ with leech microglial cells: Involvement of ClqBP-related molecule in the induction of cell chemotaxis. J Neuroinflammation 9: 37, 2012.

5. McGee AM, Douglas DL, Liang Y, Hyder SM and Baines CP: The mitochondrial protein $\mathrm{C} 1 \mathrm{qbp}$ promotes cell proliferation, migration and resistance to cell death. Cell Cycle 10: 4119-4127, 2011.

6. Dembitzer FR, Kinoshita Y, Burstein D, Phelps RG, Beasley MB, Garcia R, Harpaz N, Jaffer S, Thung SN, Unger PD, et al: gClqR expression in normal and pathologic human tissues: Differential expression in tissues of epithelial and mesenchymal origin. J Histochem Cytochem 60: 467-474, 2012.

7. Zhang X, Zhang F, Guo L, Wang Y, Zhang P, Wang R, Zhang $\mathrm{N}$ and Chen $\mathrm{R}$ : Interactome analysis reveals that $\mathrm{C} 1 \mathrm{QBP}$ (complement component 1, q subcomponent binding protein) is associated with cancer cell chemotaxis and metastasis. Mol Cell Proteomics 12: 3199-3209, 2013.

8. Rubinstein DB, Stortchevoi A, Boosalis M, Ashfaq R, Ghebrehiwet B, Peerschke EI, Calvo F and Guillaume T: Receptor for the globular heads of C1q (gC1q-R, p33, hyaluronan-binding protein) is preferentially expressed by adenocarcinoma cells. Int J Cancer 110: 741-750, 2004.

9. Chen YB, Jiang CT, Zhang GQ, Wang JS and Pang D: Increased expression of hyaluronic acid binding protein 1 is correlated with poor prognosis in patients with breast cancer. J Surg Oncol 100: 382-386, 2009.

10. Yu H, Liu Q, Xin T, Xing L, Dong G, Jiang Q, Lv Y, Song X Teng C, Huang D, et al: Elevated expression of hyaluronic acid binding protein 1 (HABP1)/P32/C1QBP is a novel indicator for lymph node and peritoneal metastasis of epithelial ovarian cancer patients. Tumour Biol 34: 3981-3987, 2013. 
11. Amamoto R, Yagi M, Song Y, Oda Y, Tsuneyoshi M, Naito S, Yokomizo A, Kuroiwa K, Tokunaga S, Kato S, et al: Mitochondrial p32/C1QBP is highly expressed in prostate cancer and is associated with shorter prostate-specific antigen relapse time after radical prostatectomy. Cancer Sci 102: 639-647, 2011

12. Itahana $\mathrm{K}$ and Zhang Y: Mitochondrial p32 is a critical mediator of ARF-induced apoptosis. Cancer Cell 13: 542-553, 2008.

13. Sunayama J, Ando Y, Itoh N, Tomiyama A, Sakurada K, Sugiyama A, Kang D, Tashiro F, Gotoh Y, Kuchino Y and Kitanaka C: Physical and functional interaction between $\mathrm{BH} 3-o n l y$ protein Hrk and mitochondrial pore-forming protein p32. Cell Death Differ 11: 771-781, 2004.

14. Meenakshi J, Anupama Goswami SK and Datta K: Constitutive expression of hyaluronan binding protein 1 (HABP1/p32/gClqR) in normal fibroblast cells perturbs its growth characteristics and induces apoptosis. Biochem Biophys Res Commun 300: 686-693, 2003

15. Kamal A and Datta K: Upregulation of hyaluronan binding protein 1 (HABP1/p32/gC1qR) is associated with Cisplatin induced apoptosis. Apoptosis 11: 861-874, 2006.

16. Hosseinimehr SJ, Nobakht R, Ghasemi A and Pourfallah TA: Radioprotective effect of mefenamic acid against radiation-induced genotoxicity in human lymphocytes. Radiat Oncol J 33: 256-260, 2015

17. Han S, Bui NT, Ho MT, Kim YM, Cho M and Shin DB: Dexamethasone inhibits TGF- $\beta 1$-induced cell migration by regulating the ERK and AKT pathways in human colon cancer cells via CYR61. Cancer Res Treat 48: 1141-1153, 2016.

18. Kim ST, Park KH, Kim JS, Shin SW and Kim YH: Impact of KRAS mutation status on outcomes in metastatic colon cancer patients without anti-epidermal growth factor receptor therapy. Cancer Res Treat 45: 55-62, 2013.

19. Lee JH, Kim KH, Park JW, Chang HJ, Kim BC, Kim SY, Kim KG, Lee ES, Kim DY, Oh JH, et al: Low-mass-ion discriminant equation: A new concept for colorectal cancer screening. Int J Cancer 134: 1844-1853, 2014.

20. Kim KH, Yeo SG, Kim WK, Kim DY, Yeo HY, Hong JP, Chang HJ, Park JW, Kim SY, Kim BC and Yoo BC: Up-regulated expression of 1-caldesmon associated with malignancy of colorectal cancer. BMC Cancer 12: 601, 2012.
21. Su F, Grijalva V, Navab K, Ganapathy E, Meriwether D, Imaizumi S, Navab M, Fogelman AM, Reddy ST and Farias-Eisner R: HDL mimetics inhibit tumor development in both induced and spontaneous mouse models of colon cancer. Mol Cancer Ther 11: 1311-1319, 2012.

22. Cho O, Hwang HS, Lee BS, Oh YT, Kim CH and Chun M: Met inactivation by $\mathrm{S}$-allylcysteine suppresses the migration and invasion of nasopharyngeal cancer cells induced by hepatocyte growth factor. Radiat Oncol J 33: 328-336, 2015

23. van Duijnhoven FJ, Bueno-De-Mesquita HB, Calligaro M, Jenab M, Pischon T, Jansen EH, Frohlich J, Ayyobi A, Overvad K, Toft-Petersen AP, et al: Blood lipid and lipoprotein concentrations and colorectal cancer risk in the European prospective investigation into cancer and nutrition. Gut 60 : 1094-1102, 2011.

24. Halley P, Kadakkuzha BM, Faghihi MA, Magistri M, Zeier Z, Khorkova O, Coito C, Hsiao J, Lawrence M and Wahlestedt C: Regulation of the apolipoprotein gene cluster by a long noncoding RNA. Cell Rep 6: 222-230, 2014.

25. Zhang X, Zhao XW, Liu DB, Han CZ, Du LL, Jing JX and Wang Y: Lipid levels in serum and cancerous tissues of colorectal cancer patients. World J Gastroenterol 20: 8646-8652, 2014.

26. Su F, Kozak KR, Imaizumi S, Gao F, Amneus MW, Grijalva V, $\mathrm{Ng} \mathrm{C}$, Wagner A, Hough G, Farias-Eisner G, et al: Apolipoprotein A-I (apoA-I) and apoA-I mimetic peptides inhibit tumor development in a mouse model of ovarian cancer. Proc Natl Acad Sci USA 107: 19997-20002, 2010.

27. Wang X, Dai S, Zhang Z, Liu L, Wang J, Xiao X, He D and Liu B: Characterization of apolipoprotein A-I as a potential biomarker for cholangiocarcinoma. Eur J Cancer Care (Engl) 18: 625-635, 2009.

28. Jiang R, Yang ZH, Luo DH, Guo L, Sun R, Chen QY, Huang PY, Qiu F, Zou X, Cao KJ, et al: Elevated apolipoprotein A-I levels are associated with favorable prognosis in metastatic nasopharyngeal carcinoma. Med Oncol 31: 80, 2014.

29. Tachibana M, Ohkura Y, Kobayashi Y, Sakamoto H, Tanaka Y, Watanabe J, Amikura K, Nishimura Y and Akagi K: Expression of apolipoprotein A1 in colonic adenocarcinoma. Anticancer Res 23: 4161-4167, 2003. 